\title{
SMART VS. INTELLIGENT VS. ACTIVE IP NETWORKS
}

\author{
Guy Pujolle \\ PRiSM - University of Versailles \\ 45, avenue des Etats-Unis - 78000 Versailles - France \\ email: Guy.Pujolle@prism.uvsq.fr
}

Abstract: IP networks have to be controlled for providing quality of service necessary for multimedia services. Three possibilities could be proposed to reach this goal:

1. The parameters of the IP architecture are controlled through a multi-agent system. In other words a meta-control is applied to the different algorithms running over the network.

2. A second possibility is to copy what was performed in telecommunication networks: a very general architecture named Intelligent Network (IN). An implementation could be seen through the TINA architecture. However, the IP Intelligent Network platform could be Java/Jini-oriented and in some sense more distributed.

3. A third possibility would deal with active networks. In this case, different possibilities are also available that will be presented. As a conclusion, the three possibilities will be compared.

\section{INTRODUCTION}

In future IP networks one of the goals is to provide a large variety of services. For almost all services the networks will need to provide explicit Quality of Service (QoS) guarantees to subscribers. Several solutions are proposed by IETF through Integrated services, Differentiated services, other flow control and signaling solutions like RSVP. However, these schemes are very difficult to optimize and do not take care of the interface between the network and the application. An intermediate level that could be named "intelligent interface" has to be provided between upper and lower layers.

The original version of this chapter was revised: The copyright line was incorrect. This has been corrected. The Erratum to this chapter is available at DOI: 10.1007/978-0-387-35581-8_35 
This software has to know what the application wants and how to control parameters of the different quality of service schemes implemented above. As a summary, one of the goals of introducing intelligence in a network is to optimize the resource but guaranteeing the service. Indeed, the intelligence to be added in the network should promote a rapid introduction of any new service with its quality of service through a flexible network architecture. This flexibility should be reached using new interfaces. Three possibilities are proposed in this paper:

- Use intelligence coming from some software agents that we could name "intelligent agents";

- Use a plate-form between the IP network and the application that looks like the Intelligent Network (IN) proposed by the telcos;

- Use the new paradigm of active networks.

The goal of this paper is to introduce these three schemes and give some comparisons.

The paper is composed as follows: intelligent agents will be introduced in section 2 . Then, in section 3 we will describe the plate-form that could be developed over an IP network to control the interface between networking elements and application entities. Section 4 provides an overview of active networks and the way these networks could help the optimization of IP networks. Finally, in section 5 we try to compare the different solutions described in previous sections to play the role of this intelligent interface necessary to control networks and applications.

\section{INTELLIGENT AGENT SYSTEMS}

A first possibility to introduce intelligence in an IP network is to implement a distributed intelligent agent system inside the different routers and terminal equipment. The goal of this distributed system is to control the application and protocol parameters and so forth to optimize resource of the network. Distributed intelligent agent systems are good candidates for this task.

First of all, let us have a look to the definition of an intelligent agent before to have them grouped to perform a function. Indeed, an intelligent agent offers a variety of definitions:

- "The term agent is used to represent two orthogonal concepts. The first is the agent's ability for autonomous execution. The second is the agent's ability to perform domain oriented reasoning" [1].

- "An agent is anything that can be viewed as perceiving its environment through sensors and acting upon that environment through effectors" [2]. 
- $\quad$ Agents are computational systems that inhabit some complex dynamic environment, sense and act autonomously in this environment, and by doing so realize a set of goals or tasks for which they are designed" [3].

- "An agent is a persistent software entity dedicated to a specific purpose" [4].

- $\quad$ Intelligent agents continuously perform three functions: perception of dynamic conditions in the environment, action to affect conditions in the environment, and reasoning to interpret perceptions, solve problems, draw inferences, and determine actions" [5].

- And so on...

Now the problem is to group these agents for providing a global task on a distributed system. The Distributed Artificial Intelligence (DAI) systems may be seen as such a grouping. The DAI field is often divided into two subareas: Distributed Problem-Solving (DPS) and Multi-Agent Systems (MAS). DPS considers how the work of solving a particular problem can be divided among a number of modules that cooperate by dividing and sharing knowledge about the problem and the developing solution. Multi-agent systems include intelligent behavior among a collection of possibly preexisting, autonomous, intelligent agents, when they can coordinate their knowledge, goals, skills, and plans to take action or solve problems [6]. The agents in a multi-agent system may be working toward a single global goal or separate individual but interacting goals [7].

Agents in MAS and DPS systems must share the knowledge about problems they face and solutions they reach, but they must also reason about the coordination processes among the agents.

DAI can be useful in domains in which action, perception, and/or control are naturally distributed. It is also a way to reproduce the human behavior when a group of experts works together to realize a task. For these reasons, multi-agent systems are envisaged to control an IP network. Several simple examples have been provided in the literature, using this kind of distributed intelligent agent systems. These systems permit a meta-control structure [8] [9]. Assuming that for each network node we associate one or several agents, the network can be seen as a multi-agent system in which the main goal is to ensure a control of the network.

The quality of service control can be seen at two levels: the local and the global level. Several agents in each node may be defined for controlling the quality of service. Each agent has to perform a specific procedure, which is triggered according to the state of the node and to the QoS required by each connection. This constitutes a local level for the congestion control. Moreover, agents can periodically interact to exchange their knowledge and ask to other agents if they need information they do not have. This constitutes the global level. 
Intelligent agents are able to acquire and to process information about situations that are "not here and not now", i.e., spatially and temporally remote. By doing so, an agent may have a chance to avoid future problems or at least to reduce the effects. These capabilities allow agents to adapt their behavior according to the traffic flows going through the node.

Finally, interruptible agents may be used. They are able to act intelligently and to respond in real time. This capability allows implementing complex mechanisms able to take care of real time decisions.

\section{IP INTELLIGENT NETWORK PLATFORM}

IP networks environment is in essence a distributed application. From this point of view, modern distributed software techniques should be applied to the development and deployment of service [10]. Distributed computing has evolved from the use of primitive messaging-passing mechanisms to Remote Procedure Call (RPC), thus providing an abstraction of the underlying communication system and location transparencies. Recently, Distributed Object Computing (DOC) adds to RPC an object-oriented architectural framework for client-server applications. The most widely adopted DOC framework is CORBA, which can be already found in several telecommunications systems, including intelligent networks, service provisioning centers, mobile communication networks, and operations systems.

The Common Object Request Broker Architecture (CORBA) [11, 12] is a standard developed by the Object Management Group (OMG), which provides a model for interactions between objects in a distributed environment. There are several international groups developing architectures for telecommunication services that use CORBA as the platform for supporting the deployment and interoperation of the control and management software. In general, they start from the Open Distributed Modeling (ODP) concepts [13], as it is the case for both the Open Distributed Management Architecture (ODMA) defined by ISO/IEC $\mathrm{JTC} 1 / \mathrm{SC} 21$ [14], and the Telecommunications Information Networking Architecture defined by the TINA Consortium (TINA-C) [15].

TINA proposes architecture for the provision and deployment of global services in such open telecommunications market. This architecture starts with the specification of an enterprise model, which identifies the roles (e.g., service and network providers, service and network managers, subscribers, and various types of users) which take part into the service, and the relationships among them. These relationships can be more complex than manager-agent paradigm, as they can involve more than two parties, and 
peer-to-peer relationships encompassing negotiation capabilities. The requirements to model these types of relationships appear when dealing with control and management of multiparty services in which different types of roles interact.

TINA plate-form, through specialized SIBs that we can name MSIB (Management SIB) and CSIB (Control SIB), is able to adapt the network as a function of the flows and the quality of service required by these flows.

\section{ACTIVE NETWORKS}

A node in a store-and-forward network examines the packet going through interpreting the different fields. This is the work of a fixed virtual machine that is able to understand the values indicated in the different fields. An active network is also a store-and-forward network but the nodes are composed of a virtual machine able to interpret a specific language. Some commands may arrive in the nodes and these orders are interpreted and perform a computation resulting in a control of the packet.

- A first problem is the arrival of these commands that either may be carried out by the packets themselves or by special packets sometimes named smart packets.

- A second problem is the language to express the command and the powerness of this language is one of the most important points. That why these networks are also called programmable networks.

- A third problem is the level of the interface the end user may command the nodes. This interface is named Application Programmable Interface (API) and is under standardization through the P1520 IEEE committee.

- A fourth problem concerns the granularity of the control. Each packet may carry a new command or just one packet may decide of the control for all other packets during a quite long time.

Let us introduce the different types of active networks (see also [16], [17], [18], [19\})

Most of the early active network architectures follow the "active packets" approach, which is fundamentally characterized by the fact that the code is carried by the packets. The nodes are also active because they allow computations up to the application layer to take place, but no active code resides in them. Therefore, the reason for calling these technologies "active packets" technologies is that active code is carried by the packets either to be executed on the data of the same packet that carries the code, or to be executed in order to change the state or the behavior of the node. Programs must be completely self-contained, thus discounting the need for persistent 
states in a router. Also, programs must fit entirely into one packet (so they cannot be more than 1 Kbyte long) and the packet should not be fragmented.

A special protocol called active network Encapsulation Protocol (ANEP) was developed for the DARPA active networks program to facilitate portability and interoperability among different active networks projects [20].

The Active IP Option [21] describes an extension to the IP options mechanism that supports the embedding of program fragments in datagrams and the evaluation of these fragments as they traverse the network. Indeed, passive packets are replaced by active capsules, which are miniature programs that are executed as they travel. These capsules can invoke predefined primitives that interact with the local node environment, and leave information behind in a node that they have visited. Subsequent capsules can carry code that depends on this information. The capsule approach is an "in-band" approach in the sense that capsules carry the code along with the data on which it operates.

In the active node approach, the packets do not carry the actual code, but instead carry some identifiers or references to predefined functions that reside in the active nodes. The packets are active in the sense that they decide which functions are going to be executed on their data, and they provide the parameters for these functions. However, the actual code resides in the active nodes, it is not carried by the packets. The motivation for such architecture comes from the active packet approach. This approach suffers from either performance-related problems because safety and security requirements are huge, or capability related problems because the only way to minimize the security and safety issues is by restricting the programs that are carried in packets $[39,40]$.

ANTS [37] is an active network toolkit where arbitrary new protocols are automatically deployed at both intermediate nodes and end systems by using mobile code techniques. The network is viewed as a distributed programming system. The architecture introduces three components/schemes: capsules, active nodes, and code distribution.

Capsules are replacements for a packet. Their function is to include a reference to the forwarding routine to be used to process the capsule at each active node. The protocols need to be executed within a restricted environment that limits their access to shared resources. Active nodes play this role. During the processing, active nodes are responsible for the integrity of the network and handle any errors that may arise.

A Java-based prototype of ANTS has been created. The security of the implementation lies in the Java system itself. The choice of Java has allowed the researchers to evolve their architecture quickly but at the cost of less control over resources usage and lower absolute performance. 


\section{COMPARISON}

The intelligent agent paradigm proposes to treat the network as multiple agent-friendly environments and the agents as programmatic entities that could move if they are mobile agents from location to location, performing tasks for users. The similarity with active networks is obvious. Indeed, many of the active network architectures use mobile code techniques that are very close to mobile software agent technology. However, the idea of active networks is much more general. Active networks visualize the network as a collection of active nodes that can perform any computations, and a collection of active packets that carry code and are indeed programs. Under that viewpoint, a mobile agent may be regarded as a specific type of an active packet, and a mobile-agent-compatible node of traditional networks could be regarded as a specific type of an active node since the latter is secure and allows any kind of computations. A fundamental difference between the two ideas is that active networks use the concept of network layer processing whereas mobile agent systems run as application programs.

Now, the plate-form solution may be viewed as an infrastructure able to interact with the end user and network protocols. This plate-form may be built using the multi-agent systems paradigm and is a general piece of software where agents may be regarded as a specific way to realize the plateform.

As a summary, the three possibilities to introduce intelligence are not really concurrent but complementary. The intelligent agent paradigm is a way to implement intelligent plate-form and active networks. Intelligent plate-form and active networks are two layers that could be assembled to form the interface between applications and the hardware architecture.

\section{CONCLUSION}

We propose three solutions to introduce some intelligence in the control of future IP high-speed networks with QoS guarantee schemes. Indeed, these three solutions are based on different concepts that could be superposed on an IP networks. A powerful solution would be the implementation of an intelligent plate-form (ODP, INCM, TINA, etc.) built with cooperative intelligent agents for controlling QoS guaranteeing processes and based on an active network controlled by the intelligent plate-form. This architecture would be based on the three concepts developed in this paper: intelligent agents, IN, and active networks. 


\section{REFERENCES}

[1] http://www.crystaliz.com/logicware/mubot.html/

[2] S. J. Russel, and P. Norvig, Artificial intelligence: a modern approach, Prentice Hall, 1995.

[3] P. Maes, Artificial life meets entertainment: life like autonomous agents, Communications of the ACM, 38, 11, pp 108-114, 1995.

[4] D.C. Smith, A. Cypher, and J. Spohrer, KidSim: programming agents without a programming language, Communications of the ACM, 37, 7, pp. 55-67, 1994.

[5] B. Hayes-Roth, An architecture for adaptive intelligent systems, Artificial Intelligent Journal, Special Issue on agents and interactivity, 72, pp329-365, 1995.

[6] E. Werner, The design of multi-agent systems, Decentralized A.I.-3, Elsevier Science Publishers B.V., 1992.

[7] A. H. Bond, and L. Gasser, eds., Readings in Distributed Artificial Intelligence, San Mateo, Calif.: Morgan Kaufmann, 1988.

[8] D. Gaïti, and G Pujolle, Performance management issues in ATM networks : traffic and congestion control, IEEE/ACM Transactions on Networking, 4, 2, 1996.

[9] D. Gaïti, and N. Boukhatem, Cooperative congestion control schemes in ATM networks, IEEE Communications Magazine, 34, 11, pp. 102-110, 1996.

[10] J. Pavón, Building telecommunications management applications with CORBA, IEEE Com Survey, 2, 1, 1999.

[11] OMG, The Common Object Request Broker: Architecture and Specification, Revision 2.2, Feb. 1998.

[12] S. Vinoski, CORBA: integrating diverse applications within distributed heterogeneous environments, IEEE Commun. Mag., 35, 2, pp. 46-55, 1997.

[13] ITU-T Recommendation X.90x: Basic Reference Model of Open Distributed Processing, ISO/IEC JTC1/SC21/WG7, Feb. 1995.

[14] Open Distributed Management Architecture, ISO/IEC JTC1/SC21 N9947, 2/1997.

[15] F. Dupuy, G. Nilsson, and Y. Inoue, The TINA Consortium: Toward Networking Telecommunications Information Services, IEEE Commun. Mag., 33, 11, pp. 78-83, 1995.

[16] D. L. Tennenhouse et al., A survey of active network research, IEEE Commun. Mag., 35, $1,1997$.

[17] IEEE Com. Magazine, Special issue on Active and Programmable Networks, xxxx 1998, vol. zz, no. yy.

[18] IEEE Network, Special issue on Active and Programmable Networks, IEEE Network, $12,3,1998$.

[19] K. Psounis, Active Networks: Applications, Security, Safety, and Architectures, IEEE Com Survey, 2, 1, 1999.

[20] D. S. Alexander et al., Active Network Encapsulation Protocol (ANEP), RFC Draft, July 1997.

[21] D. J. Wetherall and D. L. Tennenhouse, The ACTIVE_IP option, 7th ACM SIGOPS European Workshop.

[22] S. Bhattacharjee, K. L.Calvert, and E. W.Zequra, Implementation of an Active Networking Architecture, Technical Report, Georgia Institute of Technology, July 1996.

[23] S. Bhattacharjee, K. L.Calvert, and E W.Zequra, An Architecture for Active Networking, Proc. IEEE INFOCOM '97, April 1997.

[24] D. J.Wetherall, J. V. Guttag, and D. L.Tennenhouse, ANTS: A Toolkit for Building and Dynamically Deploying Network Protocols, Proc. IEEE OPENARCH '98, April 1998. 